\title{
Detachable Dissolvable Microneedles: Intra- epidermal and Intradermal Diffusion, Effect on Skin Surface, and Application in Hyperpigmentation Treatment
}

\section{Pritsana Sawutdeechaikul}

Chulalongkorn University

Silada Kanokrungsee

Srinakharinwirot University

Thanyapat Sahaspot

Srinakharinwirot University

Kamonwan Thadvibun

Srinakharinwirot University

\section{Wijit Banlunara}

Chulalongkorn University

Benchaphorn Limcharoen

Chulalongkorn University

Titiporn Sansureerungsikul

Mineed Technology

\section{Teeranut Rutwaree}

Mineed Technology

\section{Miranda Oungeun}

Chulalongkorn University

\section{Supason Wanichwecharungruang ( $\nabla$ supason.p@chula.ac.th )}

Chulalongkorn University

\section{Research Article}

Keywords: Detachable dissolvable microneedles, Intra-epidermal and intradermal diffusion,Effect on skin surface, hyperpigmentation treatment

Posted Date: September 16th, 2021

DOI: https://doi.org/10.21203/rs.3.rs-860932/v1 
License: (c) (i) This work is licensed under a Creative Commons Attribution 4.0 International License. Read Full License

Version of Record: A version of this preprint was published at Scientific Reports on December 1st, 2021. See the published version at https://doi.org/10.1038/s41598-021-03503-5. 


\section{Abstract}

Delivering bioactive compounds into skin tissue has long been a challenge. Using ex vivo porcine and rat skins, here we demonstrate that a detachable dissolvable microneedle (DDMN) array, a special dissolvable microneedle that allows needle detachment from the base within 2 min post administration, can effectively embed a model compound into epidermis and dermis. Diffusion of the compound from the needle embedding sites to the nearby skin tissue is demonstrated at various post administration periods. The relationship between the time that a conventional dissolvable microneedle array is left on skin without needle detachment from the base and the degree of skin surface abrasion at each microneedle penetration spot is also demonstrated on skin of human volunteers. Co-loading glutathione with vitamin C (vitC) can stabilize vitC in the DDMN. DDMN loaded with vitC and glutathione can help erasing post-acne-hyperpigmentation spots.

\section{Introduction}

Unlike plant and most other animals, human and a few other primates including the Indian fruit-eating bat and the guinea pig, lack L-gulono-gamma-lactone oxidase, therefore, cannot convert glucose into ascorbic acid or vitamin C (vitC). As a result, ingestion of vitC is health essential for these species.

VitC is a potent antioxidant that has been used topically in dermatology to treat and prevent changes associated with photo-aging such as wrinkle and hyperpigmentation ${ }^{1,2}$. VitC regulates cellular collagen level by acting as an essential cofactor for the actions of prolyl hydroxylase and lysyl hydroxylase in the collagen crosslinking process ${ }^{3}$. In addition, vitC directly regulates DNA involved in collagen synthesis, stabilizes procollagen messenger RNA ${ }^{4}$, and increases the synthesis of several specific lipids of the skin surface ${ }^{5}$. An in vitro experiment showed that in an absence of vitC, fibroblast cells obtained from elderly volunteers proliferated at $1 / 5$ the rate of those obtained from newborns. However, in the presence of vitC, the elderly cells proliferated better than the newborn fibroblasts. The newborn fibroblasts also proliferated four times better when exposed to vitC. Both newborn and elderly cells synthesized more collagen in the presence of vitC ${ }^{6}$. This vitamin interacts with copper ions at an active site of tyrosinase enzyme, preventing the transformation of tyrosine into melanin pigment by the enzyme, resulting in skin whitening effect ${ }^{7,8}$. Unfortunately, oral administration of vitC is not effective enough for skin hypopigmentation ${ }^{7,9}$. An ideal strategy to lessen skin hyperpigmentation is to directly supply skin tissue with vitC. Nevertheless, vitC instability in topical formulations, and limited skin penetration of the compound, must be overcome.

VitC is easily degraded by various factors such as light, oxygen, high temperature, alkali, and a presence of some heavy metals ${ }^{7}$. Reported strategies to stabilize vitC include encapsulation ${ }^{10}, \mathrm{pH}$ adjustment ${ }^{11}$, conjugation with squalene ${ }^{12}$, and inclusion of electrolytes and other antioxidants into formulations ${ }^{13}$. Nevertheless, vitC stabilization in topical formulations is still a challenge. More stable derivatives of vitC such as ascorbyl-6-palmitate, 3-0-ethyl-ascorbic acid ${ }^{14}$, magnesium ascorbyl phosphate ${ }^{15}$, ascorbyl tetraiso-palmitate ${ }^{16}$, ascorbyl glucoside ${ }^{17}$, and trisodium ascorbyl 6-palmitate 2-phosphate ${ }^{18,19}$, have 
been synthesized and used in cosmetic formulations. Unfortunately, limited stability, limited skin permeation and inefficient conversion into the active vitC in skin have been reported for these derivatives ${ }^{19,20,21}$. VitC itself demonstrates poor skin absorption when used in normal cosmetic formulations with skin compatible $\mathrm{pH}$ of $5-6$. At $\mathrm{pH}$ of less than 4 , vitC molecule is neutral because all hydroxyl groups on vitC molecule are not deprotonated, and the molecule can be more easily absorbed into the skin ${ }^{20,22}$. This, however, contradicts the recommended non-irritating $\mathrm{pH}$ range for skincare products. Controlled laser ablation of stratum corneum can improve vitC penetration into $\mathrm{skin}^{23}$, but the treatment process requires laser machine and an expert. Therefore, there is an evident interest to develop an efficient and convenient strategy to send vitC across stratum corneum into skin tissue.

The concept of microneedles (MNs) was patented in $1976^{24}$, and such array of micro-dimensioned needles was fabricated from silicon two decades later by high precision microelectronic tooling ${ }^{25}$. The device, today known as solid microneedles (solid MNs), has been used to generate skin channels prior to an application of drug solution. Dissolving MNs or DMNs are made of biocompatible/water-soluble materials that can fully dissolve in skin tissue. The drug-loaded microneedles are embedded into skin and left there for dissolution and tissue absorption. Popular materials used in DMN fabrication include hyaluronic acid (HA), gelatin, sodium alginate, poly-y-glutamic acid, polyvinylpyrrolidone (PVP) and polyvinyl alcohol $(\mathrm{PVA})^{26}$.

Despite the sound good concept of DMNs, administration of the device requires time for the dissolution of the skin-embedding needles before removing the base of the needle array. Incomplete dissolution of the embedded needles leads to the removal of undissolved part, and results in less amount of drug being delivered. In addition to these problems, production of DMNs with precise drug at only the needle part still face a few challenges including the need of centrifugation step which add cost to manufacturing, and the high drug lost during manufacturing which makes it impractical for expensive drugs. As a result, DMN has not been well accepted in medical application regardless of a recent report on a long-term safety of repeating uses of DMNs in human volunteers ${ }^{27}$.

Strategies to enable complete delivery of drug loaded DMNs into skin tissue without having to leave a DMN base on the skin for a long time, have been reported recently. Examples include putting into the needles magnesium nanoparticles that will react explosively with interstitial fluid ${ }^{28}$, creating of the DMNs with fast dissolving drug-loaded needle tips ${ }^{29}$, fabricating of needles that can swell/dissolve upon the application of electricity ${ }^{30}$, making needles to be heat responsive ${ }^{31}$, and using acid-base reaction that can generate gas at the part that connects needles to the base ${ }^{32}$.

Our lab has also reported the design and fabrication of DMNs with water penetrable backing or the so called detachable dissolvable microneedles (DDMNs) that allow quick (1-2 min) detachment of needles from the base $\mathrm{b}^{33,34,35}$.

Although it has been assumed that the drug loaded DMNs can dissolve and diffuse in skin tissue, the diffusion has never been directly demonstrated. Here via ex vivo experiments using porcine and rat skins, 
we show the dissolution and diffusion of dye molecules (used as a model drug) in epidermis and dermis at various times after their administration into the tissue via the embedment of the dye-loaded DDMNs. The relationship between the period that the microneedle array is placed on skin and the skin surface damage, was also investigated on human volunteers, and is reported here. We also show the optimization of DDMN formulation to stabilize vitC, and the use of the optimized formulation to treat facial post-acnehyperpigmentation spots in human volunteers. Effect of microneedle length on the efficacy is also reported.

\section{Results}

\section{Mechanical property and skin penetration ability of DDMNs.}

Experiments to find an additive to stabilize vitC in the DDMNs resulted in the use of glutathione at $25 \%$ of the amount of vitC (see detailed result and discussion on the additive finding in Supplementary section and Supplementary Fig. S1). We fabricated 350 and $550 \mu \mathrm{m}$ DDMNs with the square base nail-shaped needles with two loading percentages of vitC, the $20 \%$ vitC $+5 \%$ glutathione (low dose-vitC-gluta-DDMN patch) and the $40 \%$ vitC $+10 \%$ glutathione (high dose-vitC-gluta-DDMN patch), via the micro-molding technique using the mixture of hyaluronic acid (HA), polyvinyl alcohol (PVA) and sucrose at 0.5:1:1 weight ratio as needle base material (Fig. $1 \mathrm{~A}$ left and middle). Control DDMN patches (unloaded DDMNs), were also prepared. Graphs between compressive force and displaced distance of the prepared DMN patches are shown in Fig. 1B. The ex vivo skin penetration experiment was conducted using the low dosevitC-gluta-DDMN patch of 350 and $550 \mu \mathrm{m}$ needle lengths that contained red dye (Fig. 1A right) and the results are shown in Fig. $1 \mathrm{C}$.

\section{Dissolution and diffusion of microneedles in ex vivo skin tissue.}

Results of the ex vivo intra-epidermal and intradermal deliveries of $0.47 \mathrm{mg}$ of red dye by the $550 \mu \mathrm{m}$ circular DDMN patch of $1.5 \mathrm{~cm}$ diameter are shown in Fig. 2A for the rat back skin, and in Fig. 2B for the pig ear skin. Results of topical application of the solution containing the same amount of red dye to the circular area of $1.5 \mathrm{~cm}$ diameter of the ex vivo rat back and pig ear skins are also shown. The results are shown as images of the cross sectioned skin tissues at various post application times. Figure $2 \mathrm{C}$ shows layers of rat skin.

\section{In vivo skin irritation in rats.}

Skin irritation at the back of rats was tested for 1 ) the DDMN patches with needle length of $350 \mu \mathrm{m}$ containing $0.20 \mathrm{mg}$ vitC and $0.05 \mathrm{mg}$ glutathione (corresponds to $20 \%$ vitC and $5 \%$ glutathione in the needle matrix, so called low dose-vitC-gluta-350-DDMN patch), 2) the DDMN patches with needle length 
of $350 \mu \mathrm{m}$ containing $0.40 \mathrm{mg}$ vitC and $0.1 \mathrm{mg}$ glutathione (corresponds to $40 \%$ vitC and $10 \%$ glutathione in the needle matrix, so called high dose-vitC-gluta-350-DDMN patch), 3) the DDMN patches with needle length of $550 \mu \mathrm{m}$ containing $0.7 \mathrm{mg}$ vitC and $0.17 \mathrm{mg}$ glutathione (corresponds to $40 \%$ vitC and $10 \%$ glutathione in the needle matrix, so called high dose-vitC-gluta-550-DDMN patch), and 4 and 5) the unloaded DDMNs of 350 and $550 \mu \mathrm{m}$ needle lengths (so called blank-350 $\mu \mathrm{m}$ and blank-550 $\mu \mathrm{m}$ ). Skin irritation indexes and representative photographs of skin area at the tested sites (at day 1 and day 7 post the single administration), are shown in Fig. $3 \mathrm{~A}$ and $\mathrm{B}$ respectively. Histopathological findings of crosssectional views of epidermis and dermis both at day 1 and day 7 post single administration revealed no skin irritation for low dose-vitC-gluta-350-DDMNs and high dose-vitC-gluta-350-DDMNs (Fig. 3C and Supplementary Fig. S2). Only on day 1 post application, small numbers of mononuclear cells found in skin tissue treated with high dose-vitC-gluta-550-DDMNs (black arrow in Fig. 3C), but not on day 7 (Supplementary Fig. S2 D).

\section{Skin surface after administration.}

Images of the skin surface after the low dose-vitC-gluta-DDMN application over the inner arm area of human volunteers are shown in the left side of Fig. 4A and Supplementary Fig. S3 for the 350 and 550 $\mu \mathrm{m}$ needle lengths, respectively. Skin surface after the application of conventional microneedles (no needle-detaching feature, same needle morphology as the DDMNs) are shown in the right side of Fig. 4A and Supplementary Fig. S3 for the 350 and $550 \mu \mathrm{m}$ needle lengths, respectively.

\section{Post-acne-hyperpigmentation}

We tested the three types of DDMNs, unloaded DDMNs with needle length of $350 \mu \mathrm{m}$, high dose-vitCgluta-350-DDMN patch and high dose-vitC-gluta-550-DDMN patch, for their capability to erase post acne hyperpigmentation spots. Seventeen patients were enrolled, and eleven patients completed the study. Six patients could not complete the follow up due to COVID-19 pandemics situation in Bangkok. The mean age was 26.24 (SD 6.52) years old. The median of the onset post inflammatory hyperpigmentation was 3 months (IQR 1.5, 12). Here we report the result as the weekly percentages of melanin reduction (Fig. 5A) and the weekly percentages of luminance $\left(\mathrm{L}^{*}\right)$ reduction (Fig. 5B), for the 4 weeks treatment period. Representative photographs of the treated spots are shown in Fig. 5C. Slight erythema after application was found on 2 patients who were treated with high dose-vitC-gluta-550-DDMN patch. However, the erythema spontaneously disappeared within $30 \mathrm{~min}$ after the removal of the microneedle patch.

\section{Discussion}

It is well accepted that vitC is an unstable compound ${ }^{7}$. To deliver vitC into epidermis and dermis by microneedles, we first made sure that vitC did not degrade in the DDMNs. The experiments were set up to observe the degradation of the vitC and to find the compound that could effectively stabilize vitC in the DDMNs (see Supplementary information for details). It was found that vitamin E and coenzyme Q10 were 
incapable of stabilizing vitC whereas glutathione could excellently prevent vitC degradation, agreeing well with the lower standard redox potentials of glutathione $(-0.24 \mathrm{~V})$ than that of the vitC $(+0.08 \mathrm{~V})^{36}$. Coloading of glutathione with vitC (at $25 \%$ glutathione relative to the amount of vitC) into the microneedles resulted in undetectable vitC degradation for at least 6 months when kept at 25,40 or $50^{\circ} \mathrm{C}$ which corresponds to a shelf-life of at least 28 months at room temperature as estimated based on Arrhenius equation (with assumption of constant activation energy) ${ }^{37}$.

We explored the mechanical properties of the fabricated DMNs (see Fig. 1A for the DMN images). Adding vitC and glutathione to the HA-PVA-sucrose needle did not significantly change the compressive strength of the three DMNs at the low compressive force rage of 0-20 N (Fig. 1B). Since the normal force used in finger pressing microneedles into skin is around $10-20 \mathrm{~N}^{38,39,40}$, this means that skin insertion performance of the unloaded, the low dose and the high dose vitC-gluta-DMNs should remain the same. Nevertheless, the high dose vitC-gluta DMNs possess the risk of abrupt deformation at the force of $20 \mathrm{~N}$ (Fig. 1B).

After that, the ex vivo skin penetration experiment was conducted. Because vitC is colorless, some red dyes were added to the DDMNs to aid the observation (Fig. 1A). The result clearly showed that all the fabricated DDMNs (low dose- and high dose-vitC-gluta-DDMNs of both needle lengths) could effectively penetrate porcine ear skin, and all the needles were embedded in the skin tissue (Fig. 1C). Comparing to a topical application of the solution containing the same amount of hydrophilic red dye, great improvement in intra-epidermal and intradermal delivery could be achieved via DDMNs (comparing the left and the right panels in Fig. 2A and Fig. 2B). When the dye solution was topically applied onto the skin, most of the dye molecules stayed on the stratum corneum during the first 30 min post the application. Little penetration of the red dye into skin tissue via hair follicles was observe at 60 - and 120-min post application. In contrast, immediately post DDMN administration, arrays of red dye spots could be clearly observed in the skin tissue, and dissolution and diffusion of the red dye from the detached needle spots to the nearby area could be clearly observed at 30 min post administration. Within 60 min post application, even distribution of the red dye in epidermis and the upper dermis of the rat skin was observed (spanning approximately $0.5 \mathrm{~mm}$ depth from the stratum corneum) (Fig. 2A). Diffusion of dye from the upper dermis to the lower dermis took place very slowly in the rat skin. At 120 min post administration, diffusion of the dye molecules into the lower dermis was observable but majority of the dye molecules were still in the epidermis and the upper dermis. In contrast, diffusion of the hydrophilic red dye from upper dermis to the lower dermis took place more quickly in pig ear skin, i.e., even distribution of red dye in epidermis and the whole dermis ( $1 \mathrm{~mm}$ from stratum corneum) was observed at 120 min post application (Fig. 2B). The discrepancy between the rat and the pig skins was likely due to the different nature of the two skin tissues, i.e., the lipid content in rat skin is higher than in pig skin ${ }^{41}$. It should be noted here that no skin channel vestige from the microneedle penetration was observed in all cross-sectional skin tissue samples. This is likely due to the flexible and elastic nature of natural skin tissue material. 
We explored the surface of human skin after the application of both the detachable DMNs (DDMNs) and the conventional DMNs (Fig. 4A and Supplementary Fig. S3). No abrasion was observed at the area applied with $350 \mu \mathrm{m}$ detachable DMN, whereas the $550 \mu \mathrm{m}$ detachable DMN produced doubtful negligible abrasion spots that disappeared on the next observation (30 min post application). However, application of the conventional DMNs (needles not detached from the base) of both needle lengths produced obvious surface abrasion spots at needle penetration sites. For the conventional DMNs, the longer the administration time, the more severity of the skin surface abrasion appeared. Skin surface disruption was most severed on skin underwent the longest period of having the DMN patch on (120 min). Our explanation on insignificant to no surface damage after the application of the detachable type of microneedles is that the quick detaching of needles from the base automatically pushed the embedded needles down from the skin surface, allowing the tissue at the surface to reseal (Fig. 4B left). With the opening at the skin surface of less than $2 \mathrm{~min}$ in the case of detachable microneedles, elasticity and flexibility of the skin tissue material could prevent the development of skin surface hole. Unlike the detachable type of DMNs, administration of conventional DMNs involved pushing needle array into skin with the base staying on the surface and holding this position for a long time. This operation opened the skin surface at each needle penetration site for as long as the time each needle stays undissolved and attached to the base. Such application prevented the resealing of skin surface, and consequently caused abrasion spot at the needle penetrating points (Fig. 4B right). It should be noted here that for the conventional DMNs, the needles did not completely dissolve in skin tissue within $2 \mathrm{~h}$ (longest time monitored). This is likely because there was not enough water in the skin tissue to completely dissolve the DMNs.

Both the unloaded DDMNs (blank-350-DDMNs) and the 350-DDMN patch containing $190 \mu \mathrm{g}$ vitC and $47.5 \mu \mathrm{g}$ glutathione (low dose-vitC-gluta-350-DDMNs) produced no sign of irritation when tested on the back skin of rats (Fig. 3). When the amounts of vitC and glutathione were increased to $380 \mu \mathrm{g}$ vitC and 95 $\mu \mathrm{g}$ glutathione (high dose-vitC-gluta-350-DDMNs), doubtful negligible irritation (irritation index of grade $0.33 \pm 0.82$ ) was observed at day 1 post administration, but no sign of skin irritation was observed at day 7 post the single administration. Histopathological examination revealed no sign of inflammatory cell infiltration. When the length of the needles was increased to $550 \mu \mathrm{m}$, the unloaded DDMNs (blank-550DDMNs) still produced no skin irritation response. However, the 550-DDMN patch containing $664 \mu \mathrm{g}$ vitC and $166 \mu$ g glutathione (high dose-vitC-gluta-550-DDMNs) produced moderate irritation response (irritation index of grade $2 \pm 1.27$ ) at day 1 post the administration, with microscopically detectable infiltration of mononuclear cells (Fig. $3 \mathrm{C}$ ). The irritation sign and the infiltration of mononuclear cells disappeared on the next examination which was day 7. Since the blank-550-DDMNs produced no sign of skin irritation, we have concluded that the irritation observed for the high dose-vitC-gluta-550-DDMNs was likely a result of high amount of vitC and glutathione delivered into the skin.

The skin hypopigmentation efficacy of vitC-gluta-DDMNs was tested in vivo on the post acne-hyperpigmented spots on the faces of $26.24 \pm 6.52$ year-old volunteers. Daily self-application of the tested sample at the studied spot was carried out by each volunteer. The studied spot was evaluated weekly via the measurement of melanin and $L^{*}$. 
The reductions of melanin and $L^{*}$ indexes are expressed related to the values at the starting point of the same spots (before treatment). The result showed decrease in the melanin during the 4 week-tested period for all three samples. Statistically significant decrease in melanin index of the high dose vitC-gluta350-DDMNs and high dose vitC-gluta-550-DDMNs groups started at week 3 whereas that of the control group started at week 4 (Fig. 5A). The high dose vitC-gluta-350-DDMNs showed more pronounced reduction than the high dose vitC-gluta-550-DDMNs group but not at statistically significant level. The faster reduction of melanin could be attributed to the vitamin $\mathrm{C}$ and glutathione delivered into the treated skin tissue. Likewise, decrease in $L^{*}$ was observed for all three samples during the 4-week study period. Statistically significant reduction in L* for the high dose vitC-gluta-350-DDMNs group started at week 3 whereas that of the high dose vitC-gluta-550-DDMNs group and the control group started at week 4. Among the three samples, high dose vitC-gluta-350-DDMNs group gave the fastest hypopigmentation effect. We speculate that the inferior of high dose vitC-gluta-550-DDMNs group as compared to the high dose vitC-gluta-350-DDMNs group was likely a result of tiny hyperpigmented spots generated by the deep needle penetration. The pattern of the tiny hyperpigmentation spots matched the pattern of the needles (Fig. 5C red arrow). Such tiny hyperpigmentation spots were also observable in some cases for the control and the high dose vitC-gluta-350-DDMNs group, but the spots were very sporadic. We concluded that the $550 \mu \mathrm{m}$ was too deep and could cause some skin micro damage that resulted in temporary hyperpigmentation. These results agreed with the slight temporary erythema observed in two volunteers (from 17) who received the high dose vitC-gluta-550-DDMNs samples, but no erythema was observed in the other sample groups. Combining the result from the ex vivo experiment which clearly showed that diffusion could take place inside the skin tissue, the best hypopigmentation efficacy of the high dose vitCgluta-350-DDMNs group, and the micro-hyperpigmentation spots observed in the high dose vitC-gluta550-DDMNs group, we conclude that the hyperpigmentation can be treated by delivering vitC and glutathione into skin via detachable DMNs with the needle length of $350 \mu \mathrm{m}$.

With an average thicknesses of facial epidermis and dermis of $\sim 40-50 \mu \mathrm{m}$ and $\sim 1000 \mu \mathrm{m}$, respectively ${ }^{42}$, the daily administration of vitC-gluta-DDMNs of $350 \mu \mathrm{m}$ likely had the vitC delivered into epidermis and upper dermis of the facial skin (see the ex vivo result in Fig. 3), and it was indeed the targeted location for vitC to induce hypopigmentation (skin whitening). Previous studies have reported that not only the epidermal-melanocyte-keratinocyt orchestration unit but also the fibroblasts in the dermal layer play an active role in regulating skin pigmentation ${ }^{43,44,45,46}$. Since the delivered vitC likely spanned from stratum corneum to the depth of $400 \mu \mathrm{m}$ for the 350-DDMNs, the volumes of the skin tissue (administered with the $1.76 \mathrm{~cm}^{2}$ patch) saturated with the delivered vitC would be $0.704 \mathrm{~cm}^{3}$. Assuming an average density of epidermis-dermis tissue of around $0.95 \mathrm{~g} / \mathrm{cm}^{3}$, the amounts of vitC delivered into the epidermis-upper dermis would be $270 \mu \mathrm{g}$ vitC/g of tissue. Previous works have reported vitC concentration ranges in human skin of $60-640 \mu \mathrm{g}$ per $\mathrm{g}$ of fresh epidermis and 30-130 $\mu \mathrm{g}$ per $\mathrm{g}$ of fresh dermis ${ }^{1,47,48}$. From the above estimation, vitC delivered into epidermis should still be toloratable, thus explaining the no skin irritation observed for the high dose vitC-gluta-350-DDMNs group. Although, the amount of vitC delivered into the upper dermis might exceed the upper range naturally found in dermis, no irritation was observed. It is possible that the dermis can tolorate high concentration of vitC. 
The post-acne-hyperpigmentation is the result of skin tissue injuries from acne and likely is regulated by infection, inflammation and immune cells ${ }^{49,50}$. Here the DDMN treatment was carried out at the post acne period in which all the inflammation had already been cleared. Supplying vitC to the post-injured spot could help stimulating tissue regeneration and simultaneously blocking the melanogenesis, all of which led to fast generation of new tissue with less accumulation of melanin. This led to a lighter color skin appearance at the treatment spots.

\section{Methods}

\section{Fabrication of dissolvable microneedles or DMNs for mechanical testing.}

Dissolvable microneedles or DMNs were fabricated at room temperature in a clean room using the procedure as previously described ${ }^{33}$. Briefly, appropriate volume of sterile polymer solution $(2.08 \% \mathrm{HA}$ of MW 5,000 Da, 4.16\% PVA of MW 32000, 4.16\% sucrose, in weight to volume of water) were poured into silicone molds and left under the moisture control atmosphere of $5 \%$ humidity until dry. VitC-gluta-DMNs were prepared similarly except that vitC and glutathione were added to the polymer solution. DMNs of two different needle lengths were fabricated, the $350 \mu \mathrm{m}$ DMN patch $\left(1.5 \mathrm{~cm}\right.$ in diameter or $1.76 \mathrm{~cm}^{2}$ circular patch containing 89 needles positioned at the tip to tip distance of $1,120 \mu \mathrm{m}$; each needle is nailshaped with $180 \times 180 \times 200 \mu \mathrm{m}(\mathrm{W} \times \mathrm{L} \times \mathrm{H})$ square column and, $150 \mu \mathrm{m}$ height of the square pyramid on the top) and the $550 \mu \mathrm{m}$ DMN patch $\left(1.5 \mathrm{~cm}\right.$ in diameter or $1.76 \mathrm{~cm}^{2}$ circular patch containing 89 needles positioned at the tip to tip distance of $1,110 \mu \mathrm{m}$; each needle is nail-shaped with $200 \times 200 \times 400 \mu \mathrm{m}(\mathrm{W} \times$ $\mathrm{L} \times \mathrm{H}$ ) square column and, $150 \mu \mathrm{m}$ height of the square pyramid on the top). The needles were sitting on a $500 \mu \mathrm{m}$ thick base made of the same material as the needles. All prepared DMNs were examined under stereomicroscope (Olympus DP22, Tokyo, Japan).

\section{Fabrication of detachable dissolvable microneedles or DDMNs for stability experiment, ex vivo, in vivo and clinical test in volunteers}

Detachable DMNs (so called DDMNs) were fabricated using the micro-molding method in a similar fashion to the above DMN preparation except that the base of needles is attached to water penetrable membrane sheet. Briefly, for red dye-loaded DDMNs, appropriate volume of sterile polymer solution containing red dye (2.08\% HA of MW 5,000 Da, 4.16\% PVA of MW 32000, 4.16\% sucrose, $2.52 \%$ ponceau $4 \mathrm{R}, 1.56 \%$ azorubine in weight to volume of water) were poured into silicone molds and left under the moisture control atmosphere of $5 \%$ humidity until dry. Then the water penetrable membrane was attached and the DDMN patch were put under moisture control atmosphere of $2.5 \%$ humidity until completely dry. VitC-gluta-DDMNs were prepared similarly except that dye solution was replaced with vitC and glutathione solution. Two different lengths of needle of DDMN patches were fabricated, the $350 \mu \mathrm{m}$ 
DDMN patch $\left(1.5 \mathrm{~cm}\right.$ in diameter or $1.76 \mathrm{~cm}^{2}$ circular patch containing 89 needles positioned at the tip to tip distance of $1,120 \mu \mathrm{m}$; each needle is nail-shaped with $180 \times 180 \times 200 \mu \mathrm{m}(\mathrm{W} \times \mathrm{L} \times \mathrm{H})$ square column and, $150 \mu \mathrm{m}$ height of the square pyramid on the top) and the $550 \mu \mathrm{m}$ DDMN patch $(1.5 \mathrm{~cm}$ in diameter or $1.76 \mathrm{~cm}^{2}$ circular patch containing 89 needles positioned at the tip to tip distance of $1,110 \mu \mathrm{m}$; each needle is nail-shaped with $200 \times 200 \times 400 \mu \mathrm{m}(\mathrm{W} \times \mathrm{L} \times \mathrm{H})$ square column and, $150 \mu \mathrm{m}$ height of the square pyramid on the top). All prepared DDMNs were examined under stereomicroscope.

\section{Mechanical property of DMNs}

Compressive strength of DMN arrays was determined by measuring displaced distance as the tested sample was compressed at an applied axial force rate of $1 \mathrm{~mm} / \mathrm{min}$ (with the maximum force of $100 \mathrm{~N}$ or until microneedle broke) through a universal testing machine (Shimadzu EZ-S, Shimadzu Corporation, Tokyo, Japan) operated at $32{ }^{\circ} \mathrm{C}$ and a relative humidity of $7.4 \pm 2 \%$.

Tested DMN samples included 1) the DMN patch with needle length of $350 \mu \mathrm{m}$ containing $380 \mu \mathrm{g}$ vitC and $95 \mu \mathrm{g}$ glutathione (high dose-vitC-gluta-350-DMN patch which corresponds to $40 \%$ vitC and $10 \%$ glutathione in the needle matrix), 2) the DMN patch with needle length of $550 \mu \mathrm{m}$ containing $664 \mu \mathrm{g}$ vitC and $166 \mu \mathrm{g}$ glutathione (high dose-vitC-gluta-550-DMN patch which corresponds to $40 \%$ vitC and $10 \%$ glutathione in the needle matrix), 3) the DMN patch with needle length of $350 \mu \mathrm{m}$ containing $190 \mu \mathrm{g}$ vitC and $47.5 \mu$ g glutathione (low dose-vitC-gluta-350-DMN patch which corresponds to $20 \%$ vitC and $5 \%$ glutathione in the needle matrix), and 4) the DMN patch with needle length of $550 \mu \mathrm{m}$ containing $332 \mu \mathrm{g}$ vitC and $83 \mu \mathrm{g}$ glutathione (low dose-vitC-gluta-550-DMN patch which corresponds to $20 \%$ vitC and $5 \%$ glutathione in the needle matrix).

\section{Ex vivo skin penetration and diffusion}

Full thickness of fresh porcine (crossbred pig, Sus scrofa domesticus) ear skin and fresh Wistar rats (Rattus norvegicus) dorsal skin (obtained from Department of Pathology, Faculty of Veterinary Science, Chulalongkorn University, and National Laboratory Animal Center, Mahidol University, Thailand, respectively) were cleaned with water, shaved with hair clipper, and dried with tissue paper. All fresh skin pieces were used immediately.

Skin penetration of the low dose-vitC-gluta-350-DDMN patch, and the low dose-vitC-gluta-550-DDMN patch was investigated. Red dye, vitC and glutathione were co-loaded into the DDMNs. The amounts of dyes were 9.97 and $6.17 \mu \mathrm{g}$ of ponceau and azorubine in each of the low dose-vitC-gluta-350-DDMN, and 17.43 and $10.79 \mu \mathrm{g}$ of ponceau and azorubine in each of the low dose-vitC-gluta-550-DDMN patch. The experiment was initiated by pressing the DDMN patch against the stratum corneum side of the porcine skin piece with the force of $20 \mathrm{~N}$. Then the backing of the DDMNs was wet with 2 drops of water and the patch was hand-pressed against the skin for another 2 min before removing the backing from the skin. 
The skin was immediately subjected to cross sectioning and the cut surface of sectioned skin was straightaway visualized under the stereomicroscope.

Diffusion test was performed on red dye-loaded DDMNs. The red dye-loaded DDMN patch used in the experiment was the $550 \mu \mathrm{m}$ needle-height-DDMNs containing $470 \mu \mathrm{g}$ dye/patch ( $290 \mu \mathrm{g}$ ponceau and $180 \mu \mathrm{g}$ azorubine). The DDMNs were applied on the skin, then the skin pieces were placed on wet filter paper (soaked with $0.01 \mathrm{M}$ PBS buffer of $\mathrm{pH} 7.4)$. At designated times $(0,30,60,120$ min post

administration), the skin piece was surgically sectioned along the line of the embedded needle array, and the sectioned skin piece was immediately visualized under the stereomicroscope. Each control skin piece was topically applied with the red dye solution containing the same amount of dye as in each DDMN patch (470 $\mu \mathrm{g}$ dye in $20 \mu \mathrm{L}$ water spread on $1.76 \mathrm{~cm}^{2}$ area of the skin) and was processed similarly.

\section{Observation of skin surfaces of human volunteers}

The in vivo skin surface monitoring experiment on human volunteers was carried out using the low dosevitC-gluta-350-DDMN patch and the low dose-vitC-gluta-550-DDMN patch. DDMN application was carried out at the inner forearm skin of four volunteers. Each volunteer was administered with both DDMN samples ( $2 \mathrm{~min}$ application time) at location $2 \mathrm{~cm}$ apart. The skin sites were photographed by stereomicroscope at various times $(0,30,60,120 \mathrm{~min})$ post the administration. Control skin (the nearby area with no DDMN administration) was also photographed.

The same volunteer was also administered with four conventional DMNs at location $2 \mathrm{~cm}$ apart. The DMN administration was carried out with the DMN patch being left on the skin (no needle detachment feature). Each DMN patch was removed at different times, 0, 3060 and 120 min post the start of the application. Skin surface photograph was taken immediately after the removal of the DMN patch.

\section{Optimization of DDMN formulation to stabilize the loaded vitC}

Stability of vitC in solution. Three antioxidants including coenzyme Q10, vitamin E and glutathione, were experimented for their effect on vitC stability ${ }^{51,52,53}$. VitC solutions containing various amounts of each antioxidant were prepared. Final concentration of vitC was $0.002 \%(w / v)$ in water and final concentrations of antioxidants tested in the experiment were $0.002,0.001,0.0005,0.00025$ and $0.000125 \%(\mathrm{w} / \mathrm{v})$. The tested solution was kept in normal glass test tube under normal natural indoor light (UVB $(280-320 \mathrm{~nm})$ of $0.05-0.06 \mathrm{~mW} / \mathrm{cm}^{2}$ and UVA $(320-400 \mathrm{~nm})$ of $\left.3.0-5.0 \mathrm{~mW} / \mathrm{cm}^{2}\right)$, and at designated time the solution was subjected to UV-visible absorption analysis (maximum absorption of vitamin C is $265 \mathrm{~nm}$ ) using the CARY 100 Bio UV-visible spectrometer (Palo Alto, USA). Another set of the same sample was kept in the dark and analyzed similarly. 
VitC stability in DDMNs. Each of the freshly prepared DDMNs co-loaded with glutathione at $25 \%$ of the amount of vitC (low and high dose-vitC-gluta-350-DDMN patch) was kept separately in individually sealed foil package at 25 (RT), 40 and $50^{\circ} \mathrm{C}$. At specific time points, the DDMN patches were taken out and dissolved in $10 \mathrm{~mL}$ water under light-proof condition. The obtained solutions were immediately subjected to UV-visible absorption analysis as described above. At each time point, freshly prepared vitC standard solutions were used to construct calibration curve. Experiment was carried out in triplicate.

\section{In vivo skin irritancy}

All animal experiments were approved by Chulalongkorn University Institutional Animal Care and Use committee (CU-IACUC) (Protocol no.1973015). The study was carried out in compliance with the ARRIVE guidelines 2.0 checklist (Table S1) and all methods were carried out in accordance with the guidelines and CU-IACUC regulations. The small sample size was selected to minimize the numbers of animal used. Eighteen male Wistar rats (Rattus norvegicus) with the weight of 300-400 g) (Nomura Siam International Co., Ltd., Bangkok, Thailand) were housed in groups of two and given 2 weeks to acclimate to the housing environment (temperature: $22 \pm 2^{\circ} \mathrm{C}$, humidity: $50 \pm 20 \%$ and a standard $12: 12$ light: dark cycle). Eighteen, fourteen-week-old, male Wistar rats were randomly divided into 2 groups: groups of day 1 and day 7. Five types of DDMN samples were tested for skin irritation using in vivo skin at the back of rats. These groups were 1) low dose-vitC-gluta-350-DDMN patch, 2) high dose-vitC-gluta-350-DDMN patch), 3) high dose-vitC-gluta-550-DDMN patch, and 4 and 5) unloaded DDMNs of 350 and $550 \mu \mathrm{m}$ needle lengths.

Prior to DDMN administration, rats were anesthetized. The hairs on the dorsal back skin were trimmed with hair clipper (less than $1 \mathrm{~mm}$ length) and the shaved skin was cleaned and dry. DDMNs were administered (as described above) randomly by a veterinarian using double blind technique, regarding the location at the back of the rats. Gross and histopathological aspects were observed. Each animal was randomly administered with maximum 3 DDMN patches on its back.

For gross pathology, the gross lesions were assessed over the signs of skin reaction on day 1 and day 7 post single administration using the procedure of ISO 10993-10: Biological evaluation of medical devicesPart 10, Tests for irritation and skin sensitization (Draize test) and OECD 404 guideline for testing of chemicals (acute dermal irritation and corrosion). The irritation score concerning the signs of skin erythema and edema after DDMN application was calculated by dividing the sum of irritation scores of all animals in each group with the total number of animals in that group. All irritation scores, the cumulative irritation indexes, were categorized according to the following criteria: 0-0.4: negligible irritation, 0.5-1.9: slight irritation, 2-4.9: moderate irritation, 5-8: severe irritation, by 2 independent veterinary pathologists. The scoring system used for skin reaction is shown in Table S2 in the supplementary information or SI.

For histopathology, the full thickness of skins from all experimental groups on day 1 and day 7 post single administration were collected and fixed in $10 \%$ buffered formalin before processed by the routine 
hematoxylin\&eosin (H\&E) staining and observed under the light microscope by 2 independent veterinary pathologists using single blind technique. The skin samples were evaluated in terms of epidermal and dermal parameters that modified from Machtinger et al. (2004) ${ }^{54}$ and ISO 10993-10:2010.

\section{Treatment of hyperpigmentation in human}

Hypopigmentation efficacy of DDMN patches was evaluated in human volunteers through a randomized triple blind-controlled study, conducted at Srinakharinwirot skin center, Bangkok, Thailand from July 2021 to August 2021. The study was approved by the research ethic committee of Srinakharinwirot University (SWEC/F-095/2564) and was later registered in the Thai Clinical Trial Registry (TCTR20210903004) on 03/09/2021. All methods were performed in accordance with the relevant guidelines and regulations of the Declarations of Helsinki. Patients who have had allergic reaction to vitC or glutathione, history of keloid, concurrent skin infection on face, previous used of any medication and laser that have whitening effect (hydroquinone, arbutin, azelaic acid, kojic acid, licorice extract, Lakoocha extract, vitC, vitamin E, soybean extract, resorcinol, alpha/beta-hydroxy acid, nicotinamide) for 1 week before enrolled, were excluded. Seventeen patients were enrolled, and eleven patients completed the study. Six patients could not complete the follow up due to COVID-19 pandemics situation in Bangkok, Thailand. The patients were age over 18 years old who had more than 3 lesions of post inflammatory hyperpigmentation from acne on face. Informed written consent was obtained from each volunteer patient before the study.

The post inflammatory hyperpigmented lesions on the face of all patients were divided into three groups, lesion $A, B$ and $C$, by a computer-generated random sequence: lesion $A$ was treated by unloaded-350DDMNs, lesion $B$ was treated by high dose-vitC-gluta-350-DDMN patch, and lesion $C$ was treated by high dose-vitC-gluta-550-DDMN patch.

Volunteers applied the appropriated DDMNs on the designated clean and dry studied area by themselves. DDMN patch was applied daily before bedtime for 4 weeks. During the entire study period, volunteers had normal routine of skin cleansing and makeup. Personal and medical history was obtained at baseline. Colorimeter was used to assess melanin index and luminance ( $\left.L^{*}\right)$ at week 0 (baseline), 1, 2, 3 and 4. Melanin index was represented the skin darkness with scale ranging from 0 (white) to 100 (total black). The $L^{*}$ was represented skin lightness with scale ranging from 0 (total black) to 100 (white). The percentage of melanin reduction and $L$ * reduction was calculated as follows:

Percentage of melanin reduction $=[($ melanin index W0 - melanin index Wt $) /$ melanin index W0 $)] \times 100$

Percentage of $L *$ reduction $\left.=\left[\left(L^{*} W 0-L * W t\right) / L * W 0\right)\right] \times 100$

Where W0 was week 0 , and Wt was week of the measurement.

High number of the percentage of melanin reduction corresponds to large decrease in melanin.

Low number of the percentage of $L *$ reduction corresponds to large increase in skin lightening. 


\section{Statistical analysis}

The data from animal study were analyzed by one-way ANOVA followed by the Tukey's multiple comparison post hoc test using GraphPad Prism (version 5.03) software (GraphPad, USA). The data from human clinical study were analyzed by mixed linear model using STATA (version 14.0) software (Stata Corp LLC, USA). Differences were considered significant at $p<0.05$.

\section{Declarations}

\section{Acknowledgements}

This research project is supported by National Research Council of Thailand (NRCT), Center of Excellence in Materials and Bio-interfaces, Chulalongkorn University and the Second Century Fund (C2F), Chulalongkorn University, Thailand.

\section{Author contributions}

P.S. carried out the in vitro, ex vivo experiment, coordinated the project, analyzed data, and wrote the manuscript. W.B. and B.L. conducted the irritancy in rat experiments and revised the manuscript. T.S. and T.R. fabricated DDMNs, performed vitC stability tests and coordinated experiments in volunteers. M.O. fabricated DDMNs, performed vitC stability tests. S.K., T.S. and K.T. conducted the treatment of hyperpigmentation in human study. S.W. designed and coordinated the project, analyzed data, and wrote the manuscript.

\section{Additional Information}

Supplementary information accompanies this paper.

\section{Competing Interests:}

The authors declare that they have no competing interests.

\section{References}

1. Rhie, G. et al. Aging- and photoaging-dependent changes of enzymic and nonenzymic antioxidants in the epidermis and dermis of human skin in vivo. J. Invest. Dermatol, 117, 1212-1217 https://doi.org/10.1046/j.0022-202x.2001.01469.x (2001).

2. Ong, M. \& Maibach, H. in Handbook of Cosmetic Science and Technology 423-438(CRC Press, 2014). 
3. Kivirikko, K. I. \& Myllylä, R. Post-translational processing of procollagens. Ann. N. Y. Acad. Sci, 460, 187-201 https://doi.org/10.1111/j.1749-6632.1985.tb51167.x (1985).

4. Tajima, S. \& Pinnell, S. R. Ascorbic acid preferentially enhances type I and III collagen gene transcription in human skin fibroblasts. J. Dermatol. Sci, 11, 250-253 https://doi.org/10.1016/09231811(95)00640-0 (1996).

5. Uchida, Y., Behne, M., Quiec, D., Elias, P. M. \& Holleran, W. M. Vitamin C stimulates sphingolipid production and markers of barrier formation in submerged human keratinocyte cultures. J. Invest. Dermatol, 117, 1307-1313 https://doi.org/10.1046/j.0022-202x.2001.01555.x (2001).

6. Phillips, C. L., Combs, S. B. \& Pinnell, S. R. Effects of ascorbic acid on proliferation and collagen synthesis in relation to the donor age of human dermal fibroblasts. J. Invest. Dermatol, 103, 228-232 https://doi.org/10.1111/1523-1747.ep12393187 (1994).

7. Ravetti, S. et al. Ascorbic acid in skin health. Cosmetics, 6, 58-65 https://doi.org/10.3390/cosmetics6040058 (2019).

8. Ando, H., Kondoh, H., Ichihashi, M. \& Hearing, V. J. Approaches to identify inhibitors of melanin biosynthesis via the quality control of tyrosinase. J. Invest. Dermatol, 127, 751-761 https://doi.org/10.1038/sj.jid.5700683 (2007).

9. Traikovich, S. S. Use of topical ascorbic acid and its effects on photodamaged skin topography. Arch. Otolaryngol. Head Neck Surg, 125, 1091-1098 https://doi.org/10.1001/archotol.125.10.1091 (1999).

10. Yang, J. H., Lee, S. Y., Han, Y. S., Park, K. C. \& Choy, J. H. Efficient transdermal penetration and improved stability of L-ascorbic acid encapsulated in an inorganic nanocapsule. Bull. Korean Chem. Soc, 24, 499-503 https://doi.org/10.5012/bkcs.2003.24.4.499 (2003).

11. Lee, J. S. et al. The stabilization of L-ascorbic acid in aqueous solution and water-in-oil-in-water double emulsion by controlling $\mathrm{pH}$ and electrolyte concentration. J. Cosmet. Sci, 55, 1-12 (2004).

12. Gref, R. et al. Vitamin C-squalene bioconjugate promotes epidermal thickening and collagen production in human skin. Sci. Rep, 10, 16883 https://doi.org/10.1038/s41598-020-72704-1 (2020).

13. Sheraz, M., Ahmed, S., Shahnavi, I., Vaid, F. \& Iqbal, K. Formulation and stability of ascorbic acid in topical preparations. Sys. Rev. Pharm, 2, 86-90 https://doi.org/10.4103/0975-8453.86296 (2011).

14. Iliopoulos, F., Sil, B. C., Moore, D. J., Lucas, R. A. \& Lane, M. E. 3-0-ethyl---ascorbic acid: Characterisation and investigation of single solvent systems for delivery to the skin. Int. J. Pharm.: $X$, 1, 100025 https://doi.org/10.1016/j.ijpx.2019.100025 (2019).

15. Kameyama, K. et al. Inhibitory effect of magnesium l-ascorbyl-2-phosphate (VC-PMG) on melanogenesis in vitro and in vivo. J. Am. Acad. Dermatol, 34, 29-33 https://doi.org/10.1016/S0190-9622(96)90830-0 (1996).

16. Bastianini, M., Sisani, M. \& Petracci, A. Ascorbyl tetraisopalmitate inclusion into Y-cyclodextrin and mesoporous SBA-15: Preparation, characterization and in vitro release study. Cosmetics, 4, 21 https://doi.org/10.3390/cosmetics4030021 (2017). 
17. Huang, W. Y., Lee, P. C., Huang, L. K., Lu, L. P. \& Liao, W. C. Stability studies of ascorbic acid 2glucoside in cosmetic lotion using surface response methodology. Bioorg. Med. Chem. Lett, 23, 1583-1587 https://doi.org/10.1016/j.bmcl.2013.01.111 (2013).

18. Shibuya, S. et al. Topical application of trisodium ascorbyl 6-palmitate 2-phosphate actively supplies ascorbate to skin cells in an ascorbate transporter-independent manner. Nutrients, 9, 645 https://doi.org/10.3390/nu9070645 (2017).

19. Khan, H. et al. Physical and chemical stability analysis of cosmetic multiple emulsions loaded with ascorbyl palmitate and sodium ascorbyl phosphate salts. Acta Pol. Pharm, 73, 1339-1349 (2016).

20. Stamford, N. P. J. Stability, transdermal penetration, and cutaneous effects of ascorbic acid and its derivatives. J. Cosmet. Dermatol, 11, 310-317 https://doi.org/10.1111/jocd.12006 (2012).

21. Iliopoulos, F. et al. Topical delivery of 3-0-ethyl I-ascorbic acid from complex solvent systems. Sci. Pharm, 88, 19 https://doi.org/10.3390/scipharm88020019 (2020).

22. Pinnell, S. R. et al. Topical L-ascorbic acid: Percutaneous absorption studies. Dermatol. Surg, 27, 137-142 https://doi.org/10.1046/j.1524-4725.2001.00264.x (2001).

23. Lee, W. R., Shen, S. C., Kuo-Hsien, W., Hu, C. H. \& Fang, J. Y. Lasers and microdermabrasion enhance and control topical delivery of vitamin C. J. Invest. Dermatol, 121, 1118-1125 https://doi.org/10.1046/j.1523-1747.2003.12537.x (2003).

24. Gerstel, M. S. P. V. Drug Delivery Device. US patent No.3,964,482(1976).

25. Henry, S., McAllister, D. V., Allen, M. G. \& Prausnitz, M. R. Microfabricated microneedles: A novel approach to transdermal drug delivery. J. Pharm. sci, 87, 922-925 (1998). https://doi.org/10.1021/js980042+

26. Wang, M., Hu, L. \& Xu, C. Recent advances in the design of polymeric microneedles for transdermal drug delivery and biosensing. Lab Chip, 17, 1373-1387 https://doi.org/10.1039/C7LC00016B (2017).

27. Al-Kasasbeh, R. et al. Evaluation of the clinical impact of repeat application of hydrogel-forming microneedle array patches. Drug Deliv. Transl. Res, 10, 690-705 https://doi.org/10.1007/s13346020-00727-2 (2020).

28. Lopez-Ramirez, M. A. et al. Built-in active microneedle patch with enhanced autonomous drug delivery. Adv. Mater, 32, 1905740 https://doi.org/10.1002/adma.201905740 (2020).

29. Lee, H. S., Ryu, H. R., Roh, J. Y. \& Park, J. H. Bleomycin-coated microneedles for treatment of warts. Pharm. Res, 34, 101-112 https://doi.org/10.1007/s11095-016-2042-x (2017).

30. Anderson, A., Hegarty, C., Casimero, C. \& Davis, J. Electrochemically controlled dissolution of nanocarbon-cellulose acetate phthalate microneedle arrays. ACS Appl. Mater. Interfaces, 11, 3554035547 https://doi.org/10.1021/acsami.9b09674 (2019).

31. Zhang, Y. et al. Polymeric microneedles Integrated with metformin-loaded and PDA/LA-coated hollow mesoporous SiO2 for NIR-triggered transdermal delivery on diabetic rats. ACS Appl. Bio Mater, 1, 1906-1917 https://doi.org/10.1021/acsabm.8b00470 (2018). 
32. Li, W. et al. Long-acting reversible contraception by effervescent microneedle patch. Sci. Adv, 5, eaaw8145 https://doi.org/10.1126/sciadv.aaw8145 (2019).

33. Limcharoen, B. et al. Microneedle-facilitated intradermal proretinal nanoparticle delivery. Nanomaterials (Basel), 10, 368 https://doi.org/10.3390/nano10020368 (2020).

34. Pukfukdee, P. et al. Solid composite material for delivering viable cells into skin tissues via detachable dissolvable microneedles. ACS Appl. Bio Mater, 3, 4581-4589 https://doi.org/10.1021/acsabm.0c00498 (2020).

35. Wanichwecharungruang, S. \& Rutwaree, T. Dissolvable Microneedle. International patent no. Pct/th2019/000031(2018).

36. Vasdev, S., Gill, V. \& Singal, P. Modulation of oxidative stress-induced changes in hypertension and atherosclerosis by antioxidants. Exp. Clin. Cardiol, 11, 206-216 (2006).

37. ASTM F 1980-16, Standard Guide for Accelerated Aging of Sterile Barrier Systems for Medical Devices. (ASTM international 2016).

38. Davis, S. P., Landis, B. J., Adams, Z. H., Allen, M. G. \& Prausnitz, M. R. Insertion of microneedles into skin: Measurement and prediction of insertion force and needle fracture force. J. Biomech, 37, 11551163 https://doi.org/10.1016/j.jbiomech.2003.12.010 (2004).

39. Kong, X. Q., Zhou, P. \& Wu, C. W. Numerical simulation of microneedles' insertion into skin. Comput. Methods Biomech. Biomed. Engin, 14, 827-835 https://doi.org/10.1080/10255842.2010.497144 (2011).

40. Larrañeta, E. et al. A proposed model membrane and test method for microneedle insertion studies. Int. J. Pharm, 472, 65-73 https://doi.org/10.1016/j.ijpharm.2014.05.042 (2014).

41. Sato, K., Sugibayashi, K. \& Morimoto, Y. Species differences in percutaneous absorption of nicorandil. J. Pharm. Sci, 80, 104-107 https://doi.org/10.1002/jps.2600800203 (1991).

42. Chopra, K. et al. A comprehensive examination of topographic thickness of skin in the human face. Aesthet. Surg. J, 35, 1007-1013 https://doi.org/10.1093/asj/sjv079 (2015).

43. Yamaguchi, Y., Brenner, M. \& Hearing, V. J. The regulation of skin pigmentation. J. Biol. Chem, 282, 27557-27561 https://doi.org/10.1074/jbc.r700026200 (2007).

44. Yamaguchi, Y., Morita, A., Maeda, A. \& Hearing, V. J. Regulation of skin pigmentation and thickness by Dickkopf 1 (DKK1). J. Investig. Dermatol. Symp. Proc. 14, 73-75, https://doi.org/10.1038/jidsymp.2009.4 (2009).

45. Bastonini, E., Kovacs, D. \& Picardo, M. Skin pigmentation and pigmentary disorders: Focus on epidermal/dermal cross-Talk. Ann. Dermatol, 28, 279-289 https://doi.org/10.5021/ad.2016.28.3.279 (2016).

46. Kapoor, R., Dhatwalia, S. K., Kumar, R., Rani, S. \& Parsad, D. Emerging role of dermal compartment in skin pigmentation: Comprehensive review. J. Eur. Acad. Dermatol. Venereol, 34, 2757-2765 https://doi.org/10.1111/jdv.16404 (2020). 
47. Shindo, Y., Witt, E., Han, D., Epstein, W. \& Packer, L. Enzymic and non-enzymic antioxidants in epidermis and dermis of human skin. J. Invest. Dermatol, 102, 122-124 https://doi.org/10.1111/1523-1747.ep12371744 (1994).

48. Pullar, J. M., Carr, A. C. \& Vissers, M. C. M. The roles of vitamin C in skin health. Nutrients, 9 , https://doi.org/10.3390/nu9080866 (2017).

49. Koike, S. \& Yamasaki, K. Melanogenesis connection with innate immunity and toll-like receptors. Int. J. Mol. Sci, 21, 9769 https://doi.org/10.3390/ijms21249769 (2020).

50 . Wang, Z. et al. Skin microbiome promotes mast cell maturation by triggering stem cell factor production in keratinocytes. J. Allergy Clin. Immunol, 139, 1205-1216 https://doi.org/.e1206 (2017).

51. Touitou, E., Alkabes, M., Memoli, A. \& Alhaique, F. Glutathione stabilizes ascorbic acid in aqueous solution. Int. J. Pharm, 133, 85-88 https://doi.org/10.1016/0378-5173(95)04419-1 (1996).

52. Yamauchi, R. \& Vitamin, E. Mechanism of its antioxidant activity. Food Sci. Technol. Int. Tokyo, 3, 301-309 https://doi.org/10.3136/fsti9596t9798.3.301 (1997).

53. Saini, R. \& Coenzyme Q10: The essential nutrient. J. Pharm. Bioallied Sci, 3, 466-467 https://doi.org/10.4103/0975-7406.84471 (2011).

54. Machtinger, L. A. et al. Histological effects of tazarotene $0.1 \%$ cream vs. vehicle on photodamaged skin: a 6-month, multicentre, double-blind, randomized, vehicle-controlled study in patients with photodamaged facial skin. Br. J. Dermatol, 151, 1245-1252 https://doi.org/10.1111/j.13652133.2004.06186.x (2004).

\section{Figures}



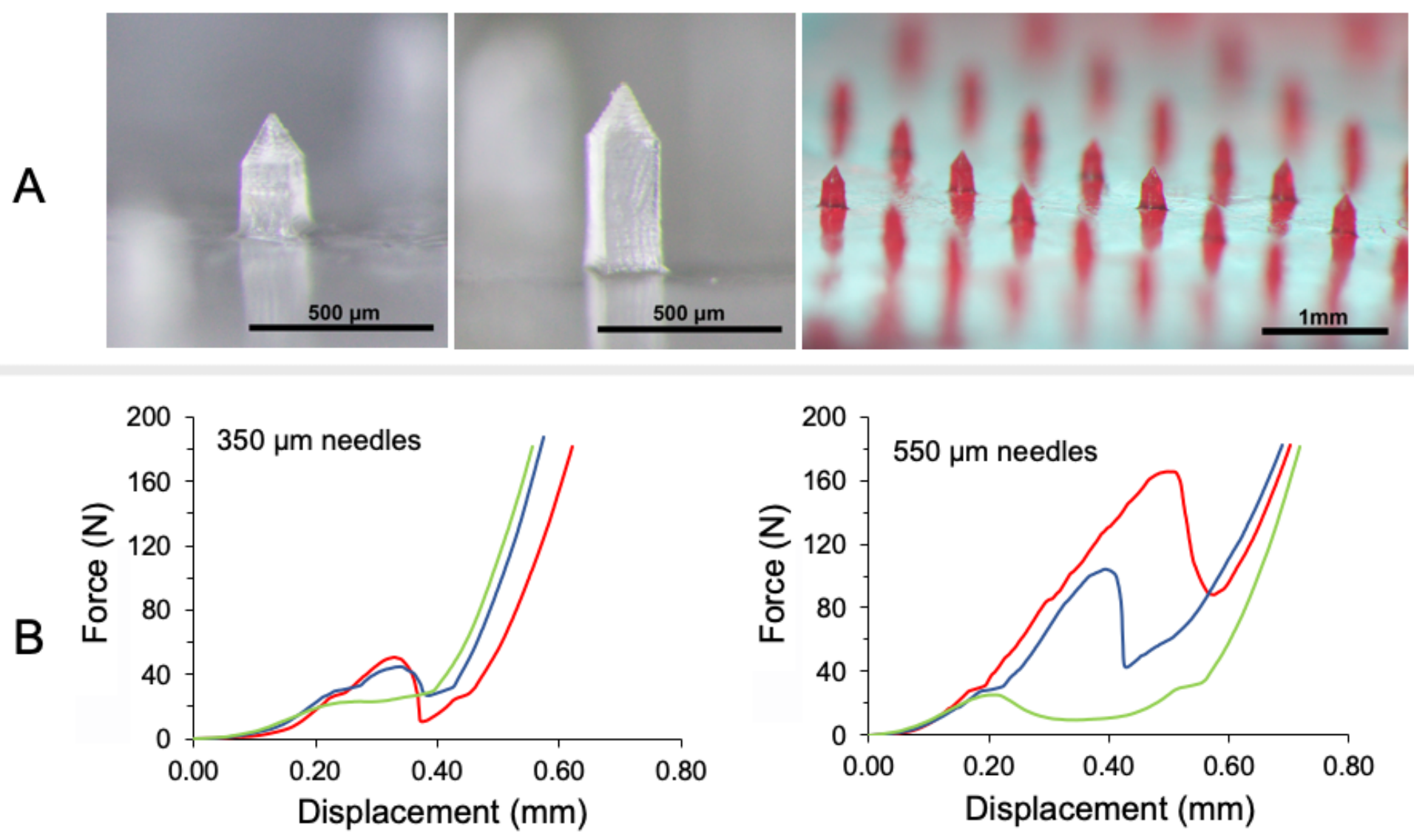

Unloaded DMNs

Low dose-vitC-Gluta-DMNs

High dose-vitC-Gluta-DMNs
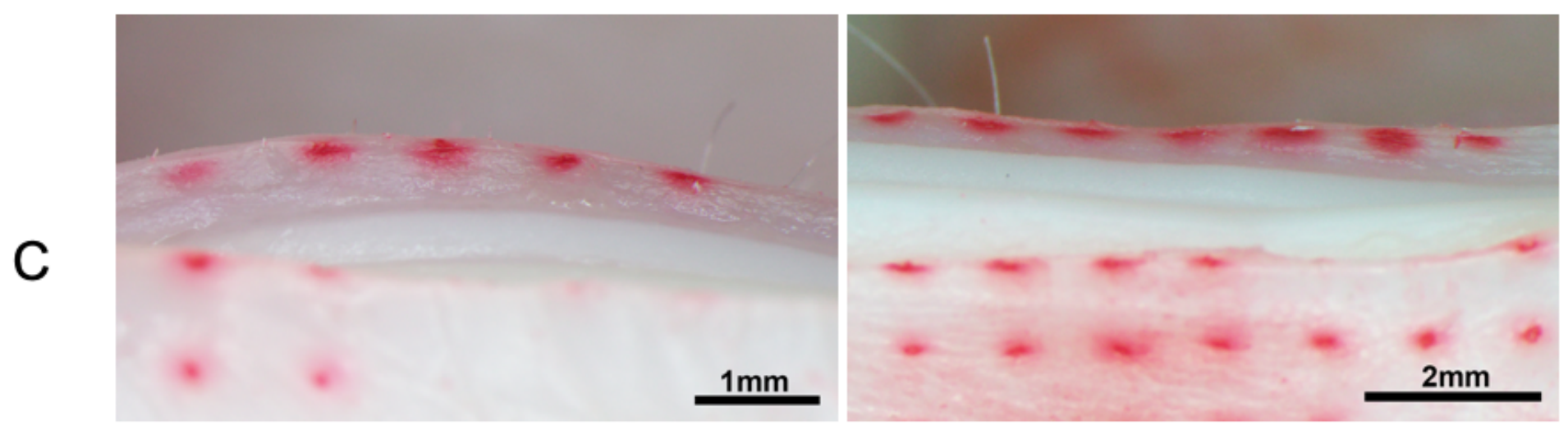

\section{Figure 1}

Morphology, mechanical property and skin penetration ability of DDMNs: A) Representative stereomicroscopic images of the low dose-vitC-gluta-DDMN of $350 \mu \mathrm{m}$ (left) and $550 \mu \mathrm{m}$ (middle) needle length and red dye-spiked low dose-vitC-gluta-DDMNs of $350 \mu \mathrm{m}$ needle length (right); B) Plotted graphs of displaced distances and applied forces for various DMNs with the needle length of $350 \mu \mathrm{m}$ (left) and $550 \mu \mathrm{m}$ (right); C) Representative stereomicroscopic images of the surgically cut porcine ear skin immediately after the administration of red dye-spiked low dose-vitC-gluta-DDMNs with the needle length of $350 \mu \mathrm{m}$ (left) and $550 \mu \mathrm{m}$ (right). 
A
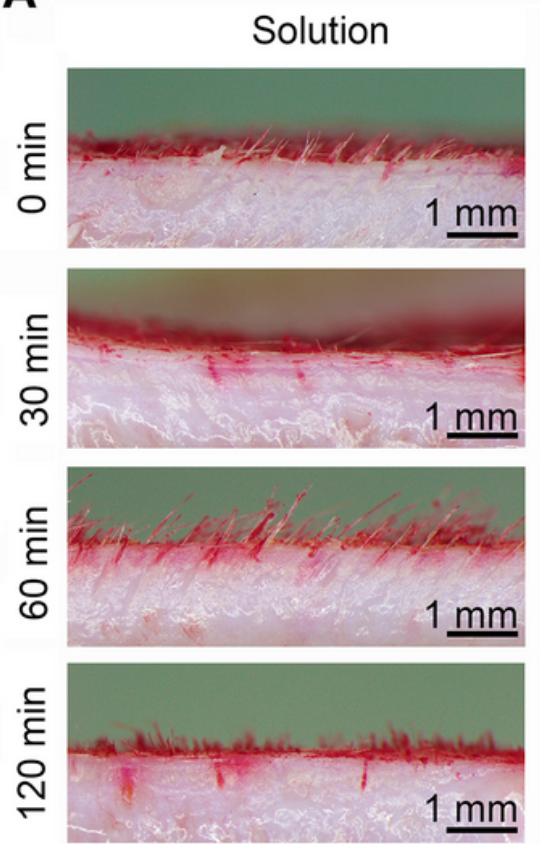

DDMNs
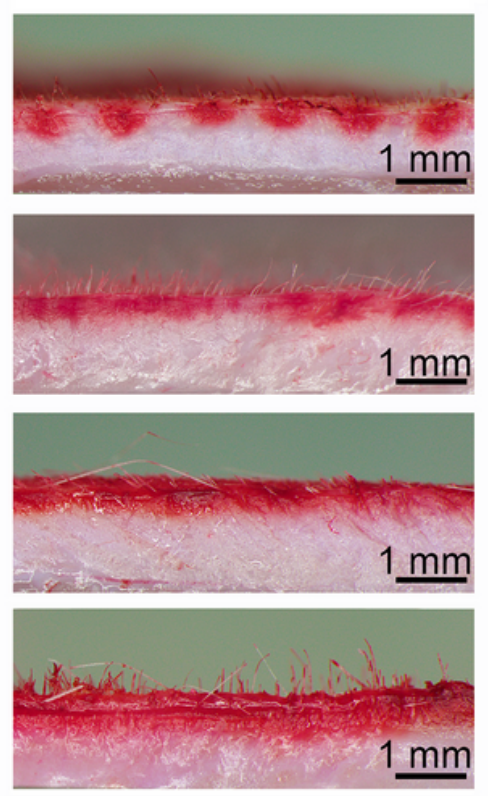

B
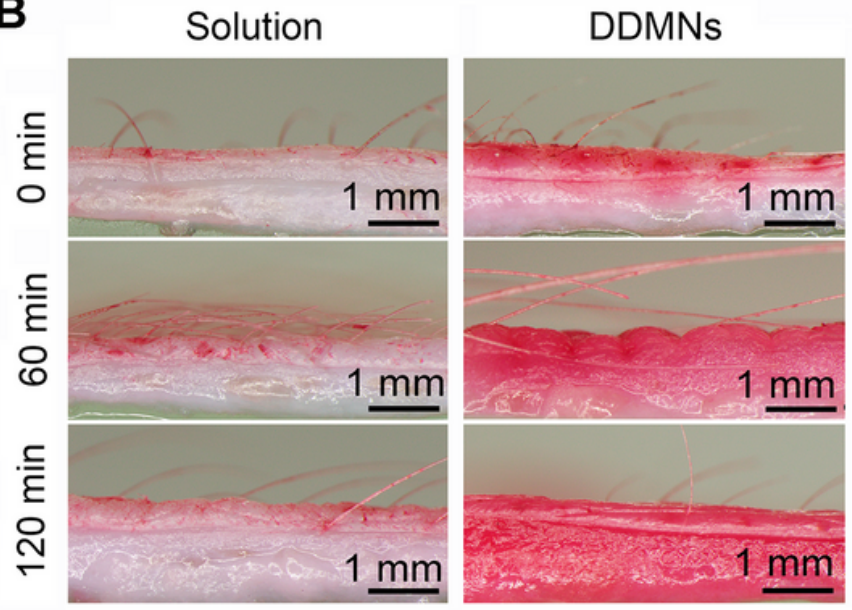

C

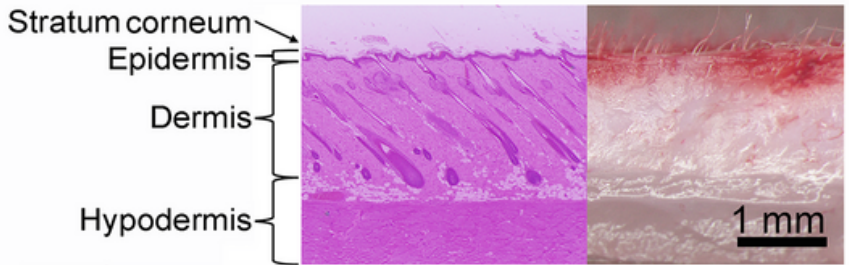

Figure 2

Stereomicroscopic images of cross sectioned skin tissues at defined times (denoted at the left side of each row) after the topical administration of red dye solution at $470 \mathrm{gg}$ dye on circular area of $1.5 \mathrm{~cm}$ diameter of the skin (left cloumn of $A$ and B) and red dye-loaded DDMNs (containing $470 \square \mathrm{g}$ dye in circular patch of $1.5 \mathrm{~cm}$ diameter , $550 \mu \mathrm{m}$ needle length) (right column of $A$ and $B$ ) for the rat back skin (A) and pig ear skin (B). Figure $C$ shows layers of rat skin. 
A

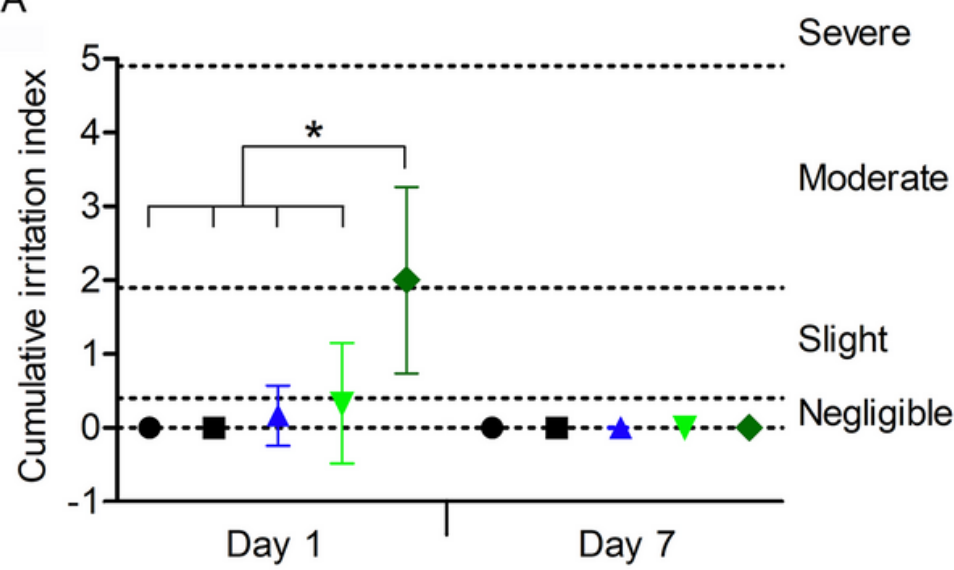

- Blank-350 $\mu \mathrm{m}$

- Blank-550 $\mu \mathrm{m}$

- Low dose-350 $\mu \mathrm{m}$

v High dose-350 um

- High dose-550 $\mu \mathrm{m}$

C

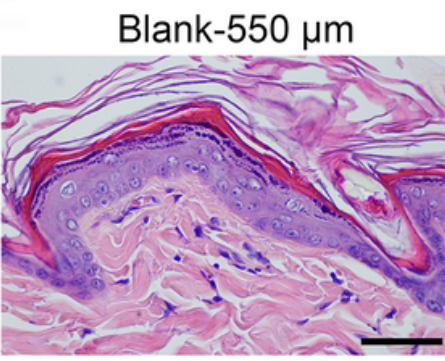

High dose-350 $\mu \mathrm{m}$

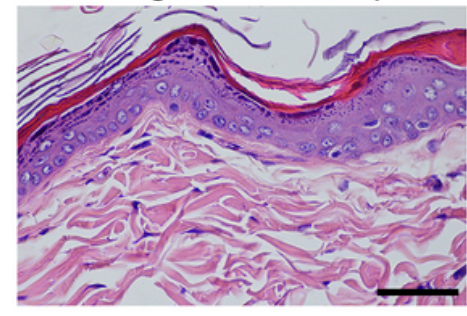

High dose-550 $\mu \mathrm{m}$

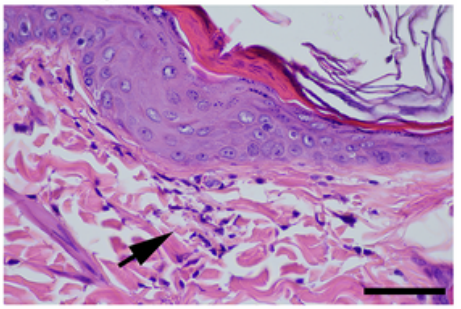

B

Day 1

Blank-350 $\mu \mathrm{m}$

Blank-550 $\mu \mathrm{m}$

Low dose-350 $\mu \mathrm{m}$

High dose-350 $\mu \mathrm{m}$

High-dose-550 $\mu \mathrm{m}$

Day 7

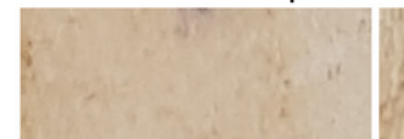

4. 310
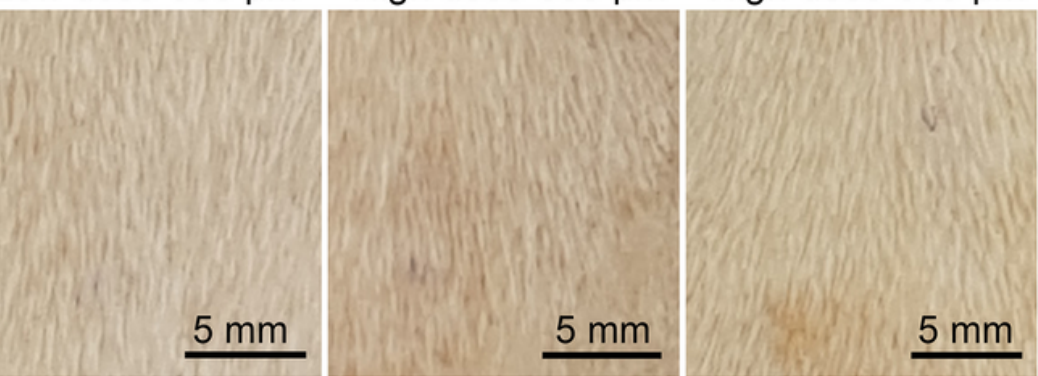

\section{Figure 3}

In vivo skin irritation. A) Cumulative irritation indexes on in vivo rat skin at day 1 and day 7 after single DDMN administration. Statistical analysis was carried out using one-way ANOVA followed by Tukey post hoc test at the significant level of $\mathrm{p}<0.05$, and * represents significant difference. Data are shown as mean with the error bars represent standard deviation obtained with $\mathrm{n}=3$ for unloaded DDMNs (Blank$350 \mu \mathrm{m}$ and Blank-550 $\mu \mathrm{m})$; and $\mathrm{n}=6$ for low dose-vitC-gluta-350-DDMNs (Low dose-350 $\mu \mathrm{m}$ ), high dosevitC-gluta-350-DDMNs (High dose-350 $\mu \mathrm{m}$ ) and high dose-vitC-gluta-550-DDMNs (high dose-550 $\mu \mathrm{m}$ ). B) Macroscopic images of skin at the back of rats at day 1 (Top row) and day 7 (Bottom row) after single DDMN administration. C) Representative pictures of the histopathology of rat skin at day 1 after the single DDMN administration. Black arrow points at mononuclear cells infiltration. Scale bar represents 50 $\mu \mathrm{m}$. 

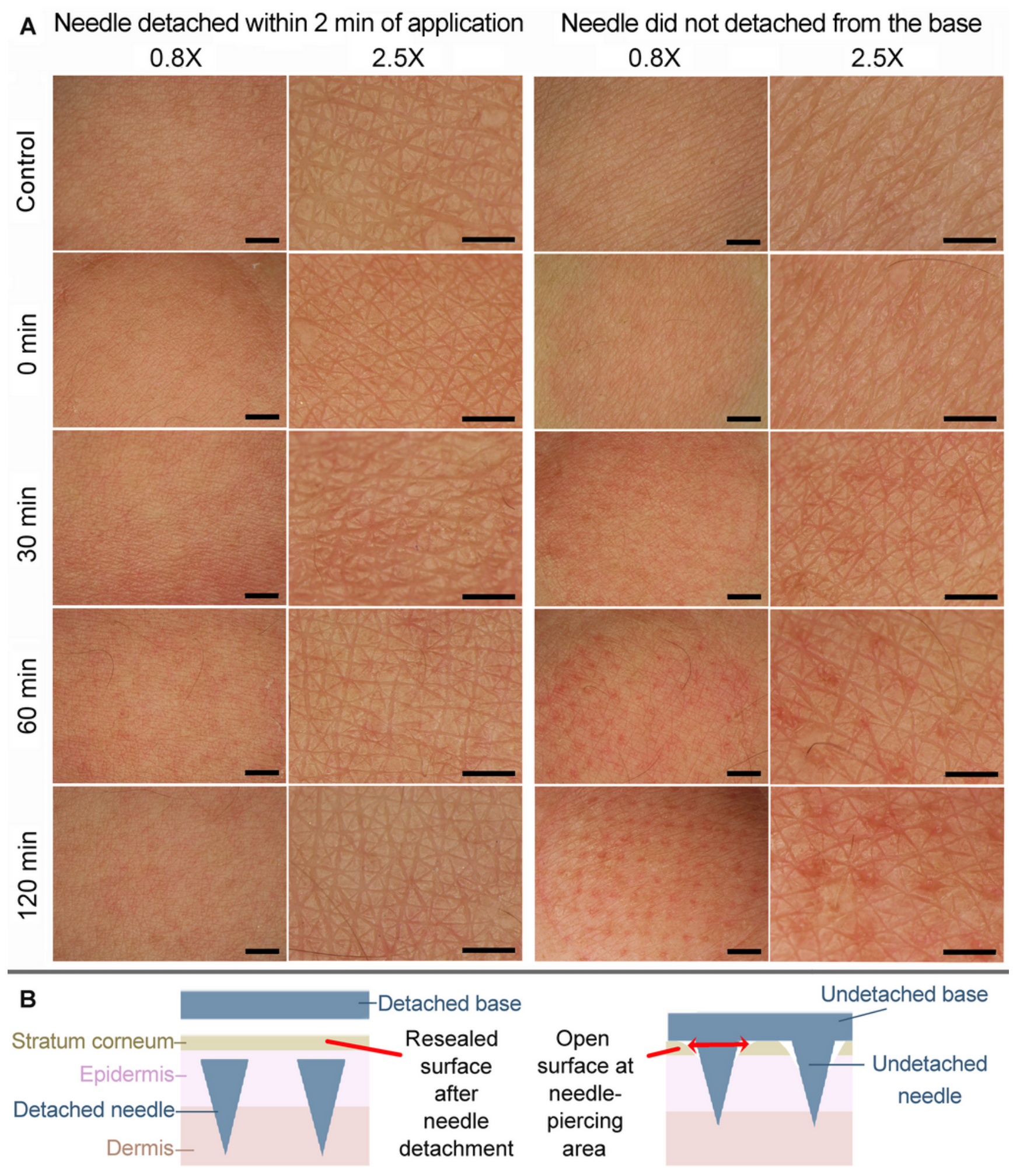

\section{Figure 4}

Skin surface conditions after microneedle administration. A) Photographs at $0.8 \mathrm{X}$ (first and third columns from the left) and 2.5X (second and fourth columns from the left) magnifications of a human volunteer inner forearm skin after the application of two types of $350 \mathrm{Im}$ microneedles, the needle-detaching type and the non-detaching type. For the DDMN or the needle-detaching type, the skin was applied with the low dose-vitC-gluta-350-DDMNs (the needles were detached and the base removed at 2 min after the 
administration), then the skin was photographed at various times post the administration (see the denoted times on the left of each row). For conventional microneedles or the DMN with no needle detaching feature, the skin was applied with the $350 \square \mathrm{m} \mathrm{DMN}$ patch (same morphology as the DDMNs) and the patch was left on the skin for 0, 30,60 and $120 \mathrm{~min}$, then the patch was removed, and the skin was photographed. Control is the image of the untreated skin. Scale bars on images in the first and the third columns from the left represent $2 \mathrm{~mm}$, and in the second and the fourth columns represent $1 \mathrm{~mm}$. B) Illustration showing how needle detachment allows for skin surface resealing (Left), and how the undetachment of needles creates skin channels at each needle penetration site (Right). 
A

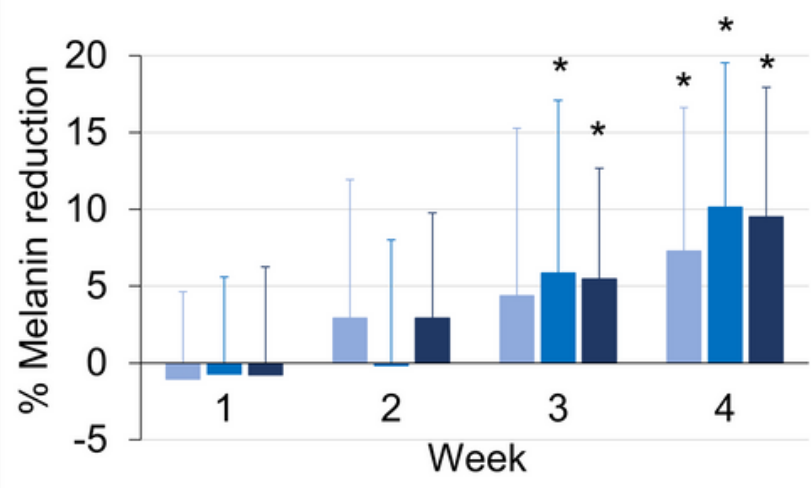

B

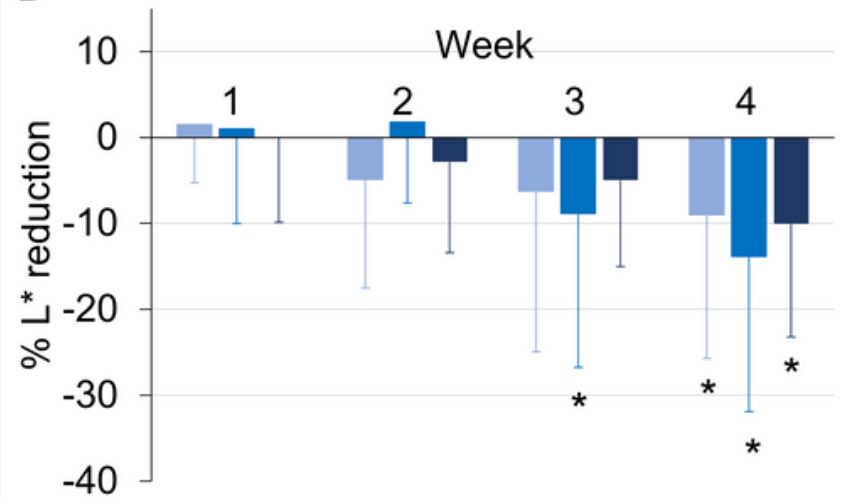

Blank-350 $\mu \mathrm{m}$

High dose-350 $\mu \mathrm{m}$

High dose-550 $\mu \mathrm{m}$

C

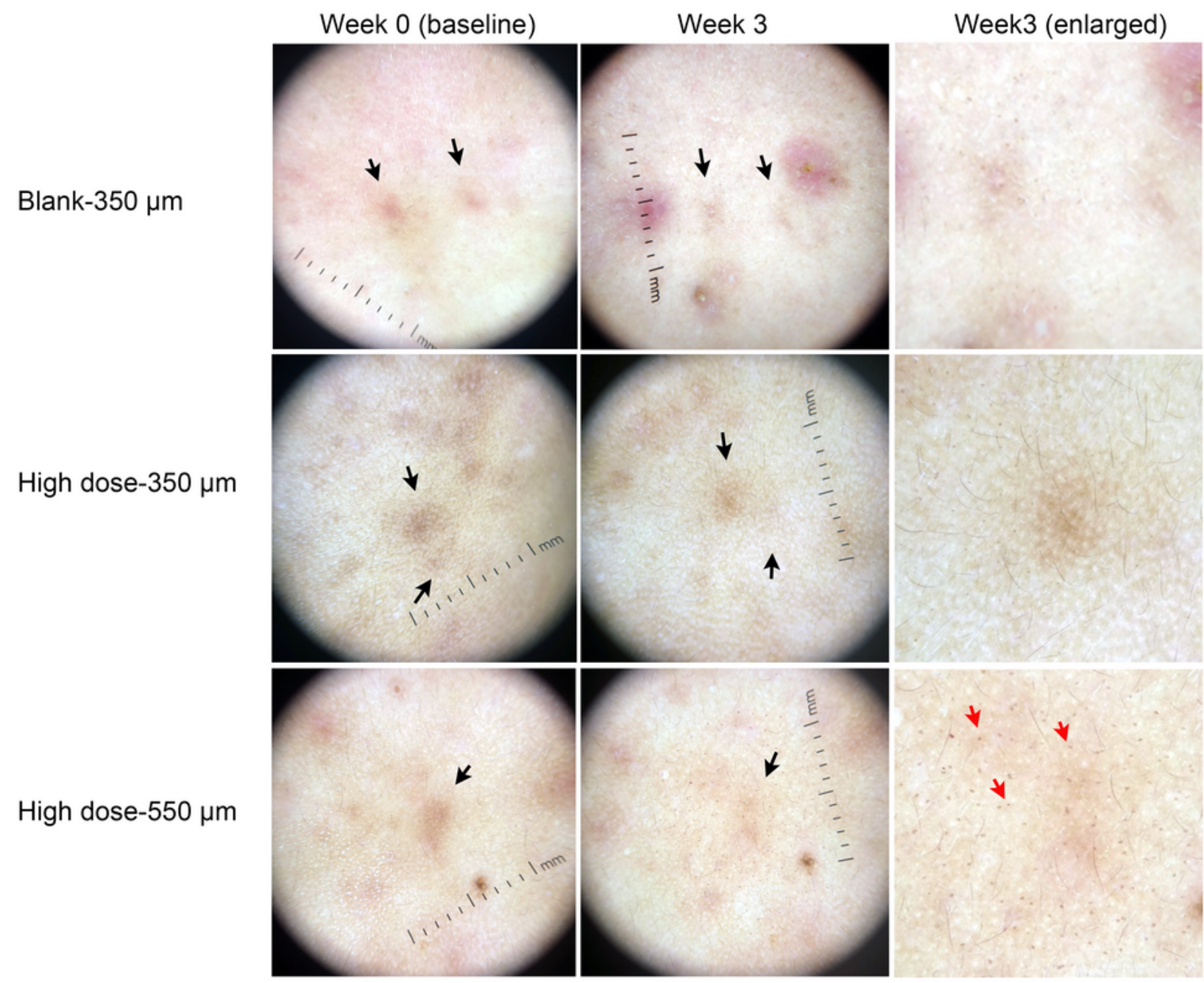

High dose-350 $\mu \mathrm{m}$

High dose-550 $\mu \mathrm{m}$

\section{Figure 5}

Hypopigmentation (skin whitening) effect of vitC-gluta-DDMNs. Hypopigmentation of the post-acnehyperpigmentation spots is reported as the percentages of melanin reduction $(A)$ and $L$ * reduction $(B)$ at various times (week 1, 2, 3 and 4) of the daily use of the three tested samples, unloaded-DDMNs (Blank$350 \mu \mathrm{m}$ ), high dose-vitC-gluta-350-DDMNs (High dose-350 $\mu \mathrm{m}$ ) and high dose-vitC-gluta-550-DDMNs (High dose-550 घm). The bar graph represents the mean value with the error bar represents standard 
deviation. Data were analyzed using mixed linear model by STATA software version 14.0 (Stata Corp LLC, USA). Significant difference between each time point and week 0 (baseline) is depicted with * at the $p<$ 0.05. C) Representative pictures of the post-acne-hyperpigmentation spots (black arrows) before the treatment (week 0 , left column) and after 3 weeks of daily application of the tested sample (week 3 , middle and right columns) are shown in $\mathrm{C}$ for the three tested samples which include unloaded-DDMNs (first row), high dose-vitC-gluta-350-DDMNs (second row) and high dose-vitC-gluta-550-DDMNs (third row). Red arrows point the hyperpigmented spots formed after the microneedle treatment.

\section{Supplementary Files}

This is a list of supplementary files associated with this preprint. Click to download.

- Supplementaryinfo.docx 